\title{
Monocyte-to-High-Density Lipoprotein Ratio Predicts the Outcome of Acute Ischemic Stroke
}

Hongbing Liu, Kai Liu, Lulu Pei, Yuan Gao, Lu Zhao, Shilei Sun, Jun Wu, Yusheng Li, Hui Fang, Bo Song and Yuming $\mathrm{Xu}$

Yuming $\mathrm{Xu}$ and Bo Song are joint senior authors.

Department of Neurology, the First Affiliated Hospital of Zhengzhou University, Henan, China

Aim: Monocyte-to-high-density lipoprotein ratio (MHR) recently emerged as an inflammatory marker and has been reported to be a novel prognostic indicator of cardiovascular diseases. However, the relationship between MHR and prognosis of acute ischemic stroke (AIS) remains unclear.

Methods: Consecutive AIS patients were prospectively identified from January 2015 to December 2017. Functional outcome was evaluated by the modified Rankin Scale (mRS). Poor outcome was defined as of mRS 3-6. Multivariate logistic regression analysis was conducted to evaluate the relationship between MHR and poor outcome.

Results: A total of 1090 AIS patients within 24 hours of the onset of symptoms were recruited. MHR was higher in poor outcome group compared to that in good outcome group [0.53 (0.37-0.69) vs. $0.48(0.33-0.60)$, $P=0.007]$. Multivariate logistic regression analysis indicated that higher MHR level was independently associated with the poor outcome at 3 months (OR 2.58, 95\% CI, 1.21-5.51, $P=0.015$ ), especially the stroke subtype of large artery atherosclerosis (OR 2.52, 95\% CI, 1.03-6.19, $P=0.034)$. Receiver operating curve (ROC) analysis showed that the area under the ROC curves for MHR was 0.67 and the best predictive cutoff value of MHR was 0.51 , with a sensitivity of $62.3 \%$ and a specificity of $66.5 \%$.

Conclusions: MHR may be a significant and independent predictor of poor functional outcome in patients with AIS.

Key words: Stroke, Prognosis, Monocytes, High-density lipoprotein, Risk factors

\section{Introduction}

Despite the improvement in antithrombotic therapy and the reinfusion strategy, the prognosis of patients with acute ischemic stroke (AIS) is still unsatisfactory ${ }^{1,2)}$. Increasing interest focuses on the identification of new prognostic markers to better enable the categorization of patients who are at higher risk for poor outcome. Recent studies have reported that inflammation plays an important role in the process of ischemic stroke ${ }^{3)}$. Some inflammatory factors, including neutrophil-to-lymphocyte ratio $(\mathrm{NLR})^{4)}, \mathrm{C}$ reactive protein $(\mathrm{CRP})^{5)}$, and $\mathrm{D}$-dimer ${ }^{6}$, have been identified through the study of AIS. Monocytes (MONO) are a type of leukocytes that play an important role in the post-ischemic inflammation ${ }^{7)}$. Monocytes were recruited to the ischemic area in the early stages of inflammatory response when AIS occurs, which promoted the damage of blood-brain barrier by releasing inflammatory mediators ${ }^{8,9)}$. In addition, high-density lipoprotein cholesterol (HDL-C) counteracts these pro-inflammatory and prooxidant effects of monocytes by preventing the migration of macrophages and oxidation of LDL molecules and by promoting the efflux of cholesterol from these cells, thus inhibiting the inflammatory process and reducing the

Address for correspondence: Yuming Xu, Department of Neurology, the First Affiliated Hospital of Zhengzhou University, No.1 Eastern Jianshe Road, Zhengzhou, Henan, China 450052. E-mail: xuyuming@zzu.edu.cn

Received: June 1, 2019 Accepted for publication: November 18, 2019

Copyright@2020 Japan Atherosclerosis Society

This article is distributed under the terms of the latest version of CC BY-NC-SA defined by the Creative Commons Attribution License. 
risk of atherosclerotic events ${ }^{10-12)}$.

Monocyte-to-HDL-C ratio (MHR), which was calculated by dividing the absolute monocyte count by the absolute HDL-C count, has emerged as a novel inflammation marker and reported to be strongly associated with cardiovascular events ${ }^{13)}$. Previous studies have shown that admission MHR values were independently correlated with increasing risk of disability or death in patients with intracerebral hemorrhage ${ }^{14)}$. Another investigation of 466 patients with AIS found that high MHR was independently correlated with a 30 -day mortality ${ }^{15)}$. Nevertheless, to our knowledge, few studies have investigated the association between MHR and function prognosis in AIS. Therefore, more prospective cohort and large sample size studies are needed to explore this relationship. It may therefore be useful to know the level of MHR to identify those at higher risk of poor outcome and to perform further potential intervention.

In this study, we aimed to evaluate the relationship between MHR and 3-month function prognosis in patients with AIS.

\section{Material and Methods}

\section{Patients and Methods}

Patients included in this study were from the database of the Henan Province Stroke Registry at the First Affiliated Hospital of Zhengzhou University ${ }^{16)}$ from January 2015 to December 2017. Ischemic stroke was diagnosed according to World Health Organization-defined criteria ${ }^{17)}$, based on clinical data, patient history, and neuroimaging results (computed tomography or magnetic resonance imaging).

Exclusion criteria were as follows: (1) time from onset to admission over 24 hours; (2) patients without complete clinical data; (3) having a history of cancer, use of an immunosuppressant, or hematologic disease; (4) severe hepatic or renal diseases; (5) major trauma, surgery, or lost to follow-up; (6) immune diseases/ chronic inflammatory diseases; and (7) chronic lung disease. This study was approved by the First Affiliated Hospital of Zhengzhou University, and informed consent was obtained from all participating patients or their immediate family members in this study.

\section{Data Collection}

Baseline data of the patients were recorded in paper-based case report forms. Demographic characteristics such as age, gender, smoking (defined as continuous or cumulative smoking $\geq 6$ months or at least 6 months every day; passive smoking refers to nonsmokers inhaling the smoke from smokers' breath for at least 15 minutes per day for more than 1 day per week), and statin use history. The stroke risk factors included history of stroke (defined as medical recordconfirmed history of ischemic, hemorrhagic stroke or subarachnoid hemorrhage), diabetes mellitus (including history of diabetes mellitus and newly diagnosed diabetes), hypertension (defined as history of hypertension and newly diagnosed hypertension), dyslipidemia (defined as low HDL-C and high triglycerides; the cutoff values were selected at HDL-C $<40 \mathrm{mg} / \mathrm{dl}$ and triglyceride $\geq 200 \mathrm{mg} / \mathrm{dl}$ in both men and women), coronary heart disease (CHD) (including myocardial infarction and angina pectoris), and atrial fibrillation. Trained neurologists assessed the baseline stroke severity of neurologic impairment within 24 hours after admission using the National Institutes of Health Stroke Scale (NIHSS).

Laboratory examinations, including blood cell counts and lipid profiles, were routinely obtained within 24 hours of fasting upon admission to the hospital. The blood cell counts and lipid profiles included white blood cell (WBC), neutrophil, MONO, lymphocyte, total cholesterol (CHO), triglycerides (TG), low-density lipoprotein cholesterol (LDL-C), and HDL-C.

\section{Follow-Up and Outcome}

The patient outcomes were evaluated by the modified Rankin Scale (mRS). Good and poor outcomes were identified by $\mathrm{mRS} \leq 2$ and $\mathrm{mRS}>2$. Clinical follow-up was completed for most patients. Follow-up results were recorded by telephone interview. Telephone interviewers were not involved in the registry and were blinded to the baseline data.

\section{Statistical Analysis}

Continuous variables were expressed as mean \pm SD or median which were analyzed by independent student's $t$-test or Mann-Whitney $U$ test. Categorical variables were presented as numbers which were analyzed using $\chi^{2}$ test or Fisher's exact test. Multivariate logistic regression analysis was conducted to assess the association between MHR and the prognosis. The receiver operating characteristic (ROC) analysis was further conducted to evaluate the ability of the MHR, MONO, and HDL to predict the outcome. Twotailed $p$ values of $<0.05$ were considered significant. All statistical analyses were performed using SPSS 19.0 software.

\section{Results}

\section{Baseline Characteristics}

A total of 1,293 AIS patients within 24 hours of the onset of symptoms were consecutively recruited 


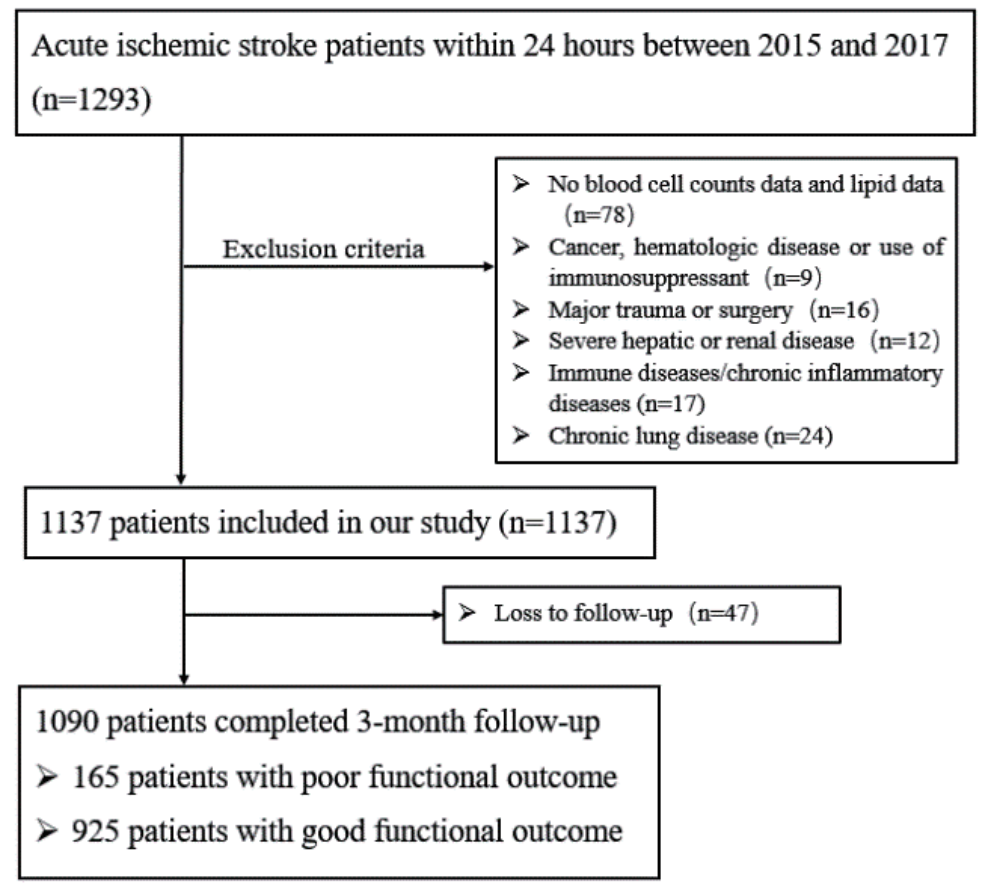

Fig. 1. Patients flowchart of the cohort

(Fig. 1), and 203 patients were excluded during the study period: 78 patients who had incomplete clinical data, 47 patients who were lost to follow-up, 62 patients who had other diseases, and 16 patients who had undergone trauma or surgery. By the time of a 3-month follow-up, 1090 patients were finally enrolled in this study. Comparison of the baseline characteristics between included and excluded patients showed no significant differences except history of hypertension (Supplemental Table 1).

During the 3-month follow-up, 925 patients had a good outcome and 165 patients had a poor outcome. The baseline clinical data of two groups are shown in Table 1. MHR [0.53 (0.37-0.69) vs. 0.48 (0.33-0.60), $P=0.007$ ] was significantly higher in the poor outcome group (Fig. 2). Compared with good outcome group, patients with a poor outcome were significantly older $(64.13 \pm 12.21$ vs. $59.32 \pm 12.64$, $P=0.001)$, were female $(38.2 \%$ vs. $31.2 \%, P=0.037)$, had higher NIHSS score at admission $(P<0.001)$, and had a prior history of smoking $(41.1 \%$ vs. $34.5 \%$, $P=0.018)$, statin use $(9.1 \%$ vs. $5.5 \%, P=0.034)$, CHD (17.2\% vs. $10.8 \%, P=0.013)$, and stroke (30.1\% vs. $20.9 \%, P=0.007)$. As for laboratory parameters, neutrophil [5.21 (4.02-6.69) vs. 4.93 (3.62-5.71), $P=0.017]$, TG [1.12 (0.88-1.70) vs. 1.35 (0.95-1.84), $P=0.012]$, HDL-C [1.03 (0.89-1.22) vs. $1.09(0.93-1.29), P=0.036]$, and MONO [0.55 $(0.43-0.67)$ vs. $0.51(0.41-0.63), P=0.027]$ reached statistical significance. However, no significant intergroup differences were observed in terms of WBC, lymphocyte, $\mathrm{CHO}$, LDL-C, and history of hypertension, dyslipidemia, and diabetes mellitus between good and poor outcome group.

Association between MHR at Admission and Functional Prognosis at 3 Months after Stroke

Multivariate logistic analysis showed that the MHR (OR 2.58, 95\% CI, 1.21-5.51, $P=0.015$ ) was independently associated with the 3-month poor outcome after the adjustment for age, gender, NIHSS score at admission, and history of stroke, atrial fibrillation, coronary heart disease, hypertension, diabetes mellitus, neutrophil, triglyceride, smoking, and statin use. The MONO (OR 2.21, 95\% CI, 1.05-4.68, $P=0.038)$ remained significantly associated, but not HDL-C (Table 2; Supplemental Table 2).

As shown in Supplemental Table 3, after multivariable adjustment, odds ratios $(95 \%$ confidence intervals) for the highest quartile of MHR were 1.64 (1.06-2.54) for primary outcome, 1.97 (0.49-2.99) for death, 3.34 (1.56-4.61) for disability, and 1.07 (1.03-1.09) for 1-U higher mRS score, compared with the lowest quartile of MHR. Every 1-SD increase of MHR level was not positively associated with poor outcomes among patients with ischemic stroke $(P=0.125)$.

TOAST subgroup analysis showed that high 
Liu et al.

Table 1. Clinical and demographic characteristics of the study population in relation to 3-month outcomes

\begin{tabular}{|c|c|c|c|}
\hline Characteristics & Good outcome $(n=925)$ & Poor outcome $(n=165)$ & $P$ Value \\
\hline Age, y & $59.32 \pm 12.64$ & $64.13 \pm 12.21$ & 0.001 \\
\hline Male & $636(68.8 \%)$ & $102(61.8 \%)$ & 0.037 \\
\hline Smoking & $315(34.5 \%)$ & $67(41.1 \%)$ & 0.018 \\
\hline Statin use history & $51(5.5 \%)$ & $15(9.1 \%)$ & 0.034 \\
\hline NIHSS score at admission & $3(2-5)$ & $8(4-12)$ & $<0.001$ \\
\hline Hypertension & $539(58.3 \%)$ & $109(65.8 \%)$ & 0.059 \\
\hline $\mathrm{CHD}$ & $99(10.8 \%)$ & $29(17.2 \%)$ & 0.013 \\
\hline Atrial fibrillation & $41(4.5 \%)$ & $19(11.5 \%)$ & 0.004 \\
\hline Diabetes mellitus & $182(19.7 \%)$ & $43(25.8 \%)$ & 0.057 \\
\hline Stroke & $192(20.9 \%)$ & $51(30.1 \%)$ & 0.007 \\
\hline Dyslipidemia & $97(10.4 \%)$ & $17(10.2 \%)$ & 0.949 \\
\hline WBC, $10^{9} / \mathrm{L}$ & $7.21(5.91-8.41)$ & $7.61(6.43-8.71)$ & 0.062 \\
\hline Neutrophil, $10^{9} / \mathrm{L}$ & $4.93(3.62-5.71)$ & $5.21(4.02-6.69)$ & 0.017 \\
\hline Lymphocyte, $10^{9} / \mathrm{L}$ & $1.73(1.41-2.14)$ & $1.71(1.11-1.97)$ & 0.059 \\
\hline MONO, $10^{9} / \mathrm{L}$ & $0.51(0.41-0.63)$ & $0.55(0.43-0.67)$ & 0.027 \\
\hline $\mathrm{CHO}, \mathrm{mmol} / \mathrm{L}$ & $4.19(3.49-4.87)$ & $4.15(3.47-4.99)$ & 0.695 \\
\hline $\mathrm{TG}, \mathrm{mmol} / \mathrm{L}$ & $1.35(0.95-1.84)$ & $1.12(0.88-1.70)$ & 0.012 \\
\hline LDL-C, mmol/L & $2.67(2.10-3.24)$ & $2.55(1.92-3.26)$ & 0.280 \\
\hline $\mathrm{HDL}-\mathrm{C}, \mathrm{mmol} / \mathrm{L}$ & $1.09(0.93-1.29)$ & $1.03(0.89-1.22)$ & 0.036 \\
\hline MHR & $0.48(0.33-0.60)$ & $0.53(0.37-0.69)$ & 0.007 \\
\hline
\end{tabular}

NIHSS, National Institutes of Health Stroke Scale; CHD, coronary heart disease; WBC, white blood cell, MONO, monocyte; $\mathrm{CHO}$, total cholesterol; TG, triglyceride; LDL-C, low-density lipoprotein; HDL-C, high-density lipoprotein and MHR, monocyte to high-density lipoprotein ratio.

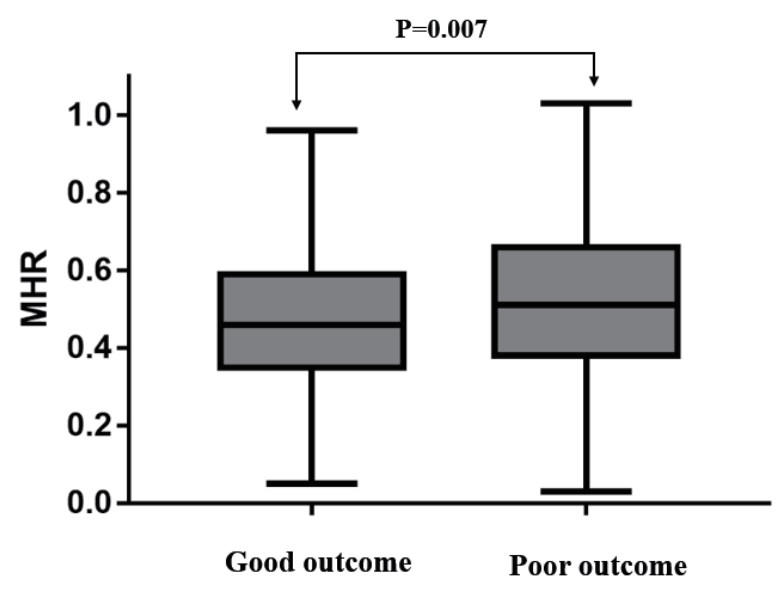

Fig. 2. Comparison of MHR between good and poor outcome in AIS patients

MHR was significantly associated with the 3-month poor outcome among stroke patients of LAA subtype (OR 2.52, 95\% CI, 1.03-6.19, $P=0.034$ ) (Table 3). There was no statistical significance for the other types.

The receiver operating characteristic analysis and area under the curve (AUC) with respect to the outcome are shown in Fig. 3. The best discriminating variable was the MHR [AUC 0.67; 95\% CI (0.64-
0.69)], which showed the highest AUC value than those of MONO [AUC 0.61; 95\% CI (0.59-0.64); $P=0.021$ ] and HDL-C [AUC 0.56; 95\% CI (0.53$0.60) ; P<0.001]$. The best predictive cutoff value was 0.51 , with a sensitivity of $62.3 \%$ and a specificity of $66.5 \%$. However, there is no significance between MONO and HDL-C counts $(P=0.106)$.

\section{Discussion}

In this study, we found that high value of MHR was associated with risks of poor outcomes within 3 months after AIS. Furthermore, the MHR represented a readily available prognostic predictor and outperformed the predictive value of the MONO and HDL-C in the current analysis.

As shown in various pathologic conditions, AIS is a local event of vascular occlusion resulting in the deprivation of oxygen and nutrients, initiating a local inflammatory immune response ${ }^{18,}{ }^{19}$. In ischemic brain damage, tissue infiltration with MONO occurs at least $72 \mathrm{~h}$ after injury onset, with the peak reaching 7 days after injury ${ }^{20,21)}$. Damaged tissue expresses chemokine receptors on the endothelial cell surfaces and interacts with leukocyte receptors, including monocyte/macrophage lineage CD $11 b+$ cells $^{222}$. Under stimulation of oxidized LDL (ox-LDL), blood mono- 
Table 2. Univariate and multivariate logistic regression analyses depicting the associations of MONO, HDL-C and MHR with 3-month outcomes of AIS

\begin{tabular}{|c|c|c|c|c|c|c|}
\hline \multirow[b]{2}{*}{ Variables } & \multicolumn{3}{|c|}{ Univariate logistic regression analysis } & \multicolumn{3}{|c|}{ Multivariate logistic regression analysis* } \\
\hline & OR & $95 \% \mathrm{CI}$ & $P$ Value & OR & $95 \%$ CI & $P$ Value \\
\hline MONO, $10^{\circ} / \mathrm{L}$ & 2.76 & $1.10-6.93$ & 0.030 & 2.21 & $1.05-4.68$ & 0.038 \\
\hline $\mathrm{HDL}-\mathrm{C}, \mathrm{mmol} / \mathrm{L}$ & 0.52 & $0.29-0.92$ & 0.045 & 0.56 & $0.28-1.09$ & 0.088 \\
\hline MHR & 2.42 & $1.20-4.87$ & 0.013 & 2.58 & $1.21-5.51$ & 0.015 \\
\hline
\end{tabular}

*Adjustment for age, gender, NIHSS score at admission, history of stroke, history of atrial fibrillation, history of coronary heart disease, history of hypertension, history of diabetes mellitus, neutrophil, triglyceride, smoking, statin use history; MONO, monocyte; HDL-C, high-density lipoprotein; MHR, monocyte to high-density lipoprotein ratio.

Table 3. Before and after adjusted odds ratio analysis between presence of high MHR in stroke subtypes

\begin{tabular}{|c|c|c|c|c|c|c|}
\hline stroke subtypes & \multicolumn{3}{|c|}{ Before adjusted odd ratio } & \multicolumn{3}{|c|}{ After adjusted odd ratio } \\
\hline Small artery disease & 0.82 & $0.67-1.45$ & 0.713 & 0.98 & $0.83-1.17$ & 0.872 \\
\hline Cardioembolic stroke & 1.01 & $0.47-1.46$ & 0.509 & 1.15 & $0.74-1.55$ & 0.815 \\
\hline Stroke of other determined etiology & 0.85 & $0.50-1.47$ & 0.568 & 1.04 & $0.88-1.67$ & 0.921 \\
\hline
\end{tabular}

*Adjustment for age, gender, NIHSS score at admission, history of stroke, history of atrial fibrillation, history of coronary heart disease, history of hypertension, history of diabetes mellitus, neutrophil, triglyceride, smoking, statin use history;

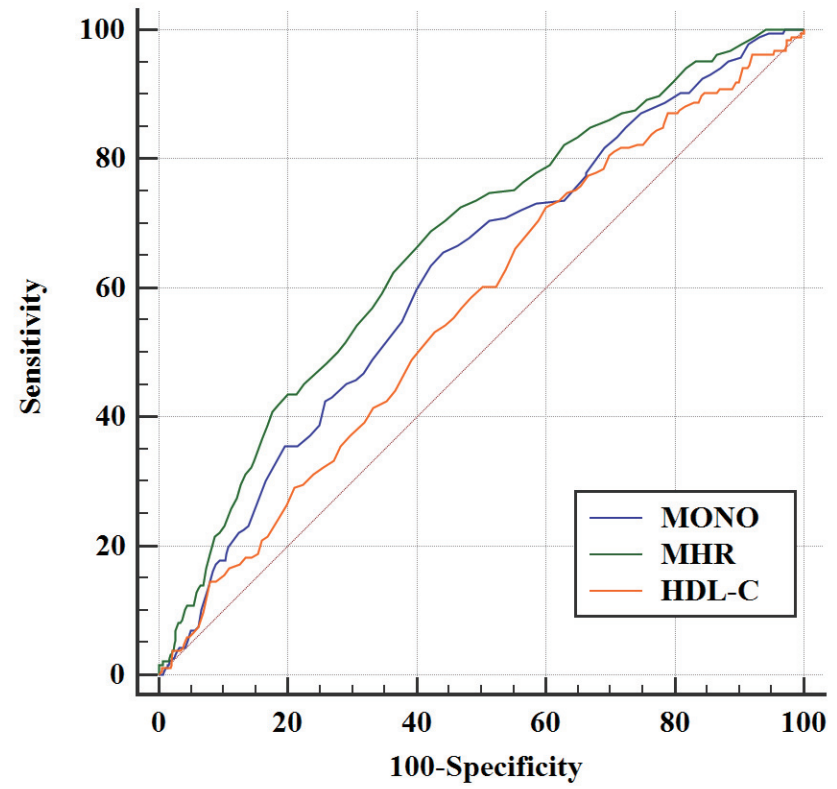

Fig. 3. Comparison of predictive power between MHR, MONO, and HDL in 3-month prognosis of patients with AIS

MHR [AUC 0.67; 95\% CI (0.64-0.69)] showed the highest AUC value than those of MONO [AUC 0.61; 95\% CI (0.59-0.64); $p=0.021$ ] and HDL-C [AUC 0.56; 95\% CI (0.53-0.60); $p<$ $0.001]$ cytes adhered to endothelial cells differentiated into macrophages. Macrophages not only gobble up oxLDL and turn into foam cells but also secrete interleukin-1 (IL-1 $\beta$ ), tumor necrosis factor- $\alpha$ (TNF- $\alpha$ ), and other inflammatory factors ${ }^{23,24)}$. Animal studies have demonstrated the association of monocyte count with the extent of inflammation. Qiao et al. ${ }^{25)}$, who investigated inflammation in macrophage colony-stimulating factor-deficient mice, reported a gene dosage-related reduction in atherosclerosis correlated with a decrease in blood monocyte counts. In clinical studies, monocyte activation was also observed in peripheral blood after stroke, especially during the acute phase of ischemic stroke ${ }^{26)}$. A study from Europe reported that baseline monocytes positively correlated with stroke severity and disability across all time points in a 90-day follow-up ${ }^{27)}$. Another study showed that a number of monocytes and plasma MCP-1 level could be clinical prognostic biomarkers as early indicator of severity of illness in patients with AIS ${ }^{28)}$. These are consistent with our findings. On the other hand, HDL-C represents well-known anti-inflammatory and antioxidant as well as antithrombotic effects; these activities have been shown in accordance with both the quality and quantity of HDL- $\mathrm{C}^{29,30)}$. Moreover, it has been confirmed that HDL-C particles exhibited anti-inflammatory effects on human monocytes by inhibiting activation of CD11 $\mathrm{b}^{31,32)}$. Other studies have shown that the inhibitory effect of HDL-C may 
be related to the signaling pathway mediated by scavenger receptor class B type I and sphingosine-1-phosphate receptor $^{7,11)}$.

Recently, the MHR as a novel marker to reflect inflammation was relatively more stable than single blood parameters ${ }^{33}{ }^{34}$; ; therefore, it may be more valuable than monocyte or HDL-C counts alone in the prediction of various diseases including cardiovascular and cerebrovascular diseases. Several studies reported that high MHR had a linear association with the rates of cardiovascular events and mortality. The pioneering study of Kanbay et al. ${ }^{35)}$ investigated that higher MHR has been associated with worse cardiovascular prognosis and independent predictor of major cardiovascular events in chronic kidney disease. Following this study, Shoujiang You ${ }^{36)}$ reported this marker in acute intracerebral hemorrhage population and suggested that MHR was independently associated with disability or death at hospital discharge and at 3 months post-stroke. A study by Asli Bolayir ${ }^{15)}$ enrolled 466 patients who were referred to clinic within the first 24 hours of symptom presentation and diagnosed with AIS; the findings demonstrated that high MHR was independently correlated with a 30-day mortality. Another investigation of 3,798 Chinese patients scheduled for selective coronary angiography found that MHR, as a novel inflammation-based marker, seemed to be an independent predictor of severity of coronary artery disease and future cardiovascular events in patients with $\mathrm{ACS}^{37)}$. However, to our knowledge, few studies have investigated the association between MHR and prognosis in AIS. Therefore, more prospective cohort and large sample size studies are needed to further confirm this relationship.

In addition, the etiological mechanism of different subtypes AIS is different, which is important for the treatment and evaluation of prognosis. The etiological mechanism of large artery atherosclerosis (LAA) stroke is atherosclerosis ${ }^{38}$. MHR, as an inflammatory factor, may play a role in cell proliferation, macrophage chemotaxis, neutrophil exudation, oxidative stress, and other processes ${ }^{39)}$. MHR may promote the formation of foam cells by activating the release of macrophage inflammatory mediators and may also affect the formation and development of atherosclerotic plaques by inhibiting the adhesion of smooth muscle cells to the matrix and other processes ${ }^{39,40)}$. In this study, high MHR level was associated with all stroke subtypes, especially the stroke subtype of large artery atherosclerosis. So far, there has been no study on the relationship between MHR and prognosis of different subtypes of stroke. We hypothesize that MHR may not equally contribute to subsequent stroke events among stroke patients of LAA and other subtypes.

There were several limitations in our study. First, our study is a single center and selection bias was unavoidable. Second, MONO and HDL-C concentrations were only collected once; thus there was a lack of dynamic data. Finally, some important inflammatory factors, including $\mathrm{C}$ reactive protein, procalcitonin, and erythrocyte sedimentation rate, were not routinely screened among included patients. For these reasons, more detailed and extensive prospective studies are required in the future.

\section{Conclusion}

In summary, our findings suggested that MHR may be a significant and independent predictor of unfavorable functional outcome in patients with AIS.

\section{Acknowledgments}

The study was supported by the National Nature Science Foundation of China (8160051522 and 52111000 to Dr. Bo Song).

\section{Conflict of Interest}

The authors declare that they have no conflict of interest.

\section{Author Contribution}

Project development: Dr. Yuming Xu and Dr. Bo Song; Writing (original draft preparation): Dr. Hongbing Liu; Data analysis: Dr. Lu Zhao; Dr. Hui Fang and Dr. Bo Song; English editing help: Dr. Kai Liu; Dr. Yuan Gao; Data collection: Dr. Hongbing Liu; Dr. Lulu Pei; Figures and Tables: Dr. Yusheng Li; Dr. Shilei Sun and Dr. Jun Wu;

\section{Ethical Approval}

All procedures performed in studies involving human participants were in accordance with the ethical standards of the institutional and/or national research committee and with the 1964 Declaration of Helsinki and its later amendments or comparable ethical standards.

\section{References}

1) Zerna C, Thomalla G, Campbell B C V, Rha J H, Hill M D. Current practice and future directions in the diagnosis and acute treatment of ischaemic stroke. Lancet, 2018; 392: $1247-1256$ 
2) Pandian J D, Gall S L, Kate M P, Silva G S, Akinyemi R O, Ovbiagele B I, Lavados P M, Gandhi D B C, Thrift A G. Prevention of stroke: a global perspective. Lancet, 2018; 392: 1269-1278

3) Kim J Y, Park J, Chang J Y, Kim S H, Lee J E. Inflammation after Ischemic Stroke: The Role of Leukocytes and Glial Cells. Exp Neurobiol, 2016; 25: 241-251

4) Kocaturk O, Besli F, Gungoren F, Kocaturk M, Tanriverdi $Z$. The relationship among neutrophil to lymphocyte ratio, stroke territory, and 3-month mortality in patients with acute ischemic stroke. Neurol Sci, 2019; 40: 139146

5) Kitagawa K, Hosomi N, Nagai $Y$, Kagimura T, Ohtsuki T, Maruyama $\mathrm{H}$, Origasa $\mathrm{H}$, Minematsu K, Uchiyama $S$, Nakamura M, Matsumoto M, Collaborators J S. Cumulative Effects of LDL Cholesterol and CRP Levels on Recurrent Stroke and TIA. J Atheroscler Thromb, 2019; 26: 432-441

6) Hamatani Y, Nagai T, Nakai M, Nishimura K, Honda Y, Nakano H, Honda S, Iwakami N, Sugano Y, Asaumi Y, Aiba T, Noguchi T, Kusano K, Toyoda K, Yasuda S, Yokoyama H, Ogawa H, Anzai T, Na D E F I. Elevated Plasma D-Dimer Level Is Associated With Short-Term Risk of Ischemic Stroke in Patients With Acute Heart Failure. Stroke, 2018; 49: 1737-1740

7) Urra X, Villamor N, Amaro S, Gomez-Choco M, Obach V, Oleaga L, Planas A M, Chamorro A. Monocyte subtypes predict clinical course and prognosis in human stroke. J Cereb Blood Flow Metab, 2009; 29: 994-1002

8) Tellides G, Pober J S. Inflammatory and immune responses in the arterial media. Circ Res, 2015; 116: 312322

9) Wang C, Petriello M C, Zhu B, Hennig B. PCB 126 induces monocyte/macrophage polarization and inflammation through AhR and NF-kappaB pathways. Toxicol Appl Pharmacol, 2019; 367: 71-81

10) Ebtehaj S, Gruppen E G, Parvizi M, Tietge U J F, Dullaart R P F. The anti-inflammatory function of HDL is impaired in type 2 diabetes: role of hyperglycemia, paraoxonase- 1 and low grade inflammation. Cardiovasc Diabetol, 2017; 16: 132

11) Feingold K R, Grunfeld C. Effect of inflammation on HDL structure and function. Curr Opin Lipidol, 2016; 27: $521-530$

12) Eren E, Ellidag H Y, Aydin O, Yilmaz N. HDL functionality and crystal-based sterile inflammation in atherosclerosis. Clin Chim Acta, 2015; 439: 18-23

13) Kanbay M, Solak Y, Unal H U, Kurt Y G, Gok M, Cetinkaya H, Karaman M, Oguz Y, Eyileten T, Vural A, Covic A, Goldsmith D, Turak O, Yilmaz M I. Monocyte count/HDL cholesterol ratio and cardiovascular events in patients with chronic kidney disease. Int Urol Nephrol, 2014; 46: 1619-1625

14) You S, Zhong C, Zheng D, Xu J, Zhang X, Liu H, Zhang Y, Shi J, Huang Z, Cao Y, Liu C F. Monocyte to HDL cholesterol ratio is associated with discharge and 3-month outcome in patients with acute intracerebral hemorrhage. J Neurol Sci, 2017; 372: 157-161

15) Perillaud-Dubois C, Pilmis B, Diep J, De Ponfilly G P, Perreau S, Ruffier D'epenoux L, Mizrahi A, Couzigou C, Vidal B, Le Monnier A, Nguyen Van J C. Performance of rapid antimicrobial susceptibility testing by disk diffusion on MHR-SIR agar directly on urine specimens. Eur J Clin Microbiol Infect Dis, 2019; 38: 185-189

16) Zhao L, Wang R, Song B, Tan S, Gao Y, Fang H, Lu J, $\mathrm{Xu}$ Y. Association between atherogenic dyslipidemia and recurrent stroke risk in patients with different subtypes of ischemic stroke. Int J Stroke, 2015; 10: 752-758

17) Stroke--1989. Recommendations on stroke prevention, diagnosis, and therapy. Report of the WHO Task Force on Stroke and other Cerebrovascular Disorders. Stroke, 1989; 20: 1407-1431

18) Shim R, Wong $\mathrm{C} \mathrm{H}$. Ischemia, Immunosuppression and Infection--Tackling the Predicaments of Post-Stroke Complications. Int J Mol Sci, 2016; 17

19) Collaborators G B D S. Global, regional, and national burden of stroke, 1990-2016: a systematic analysis for the Global Burden of Disease Study 2016. Lancet Neurol, 2019; 18: 439-458

20) Ajami B, Bennett J L, Krieger C, Tetzlaff W, Rossi F M. Local self-renewal can sustain CNS microglia maintenance and function throughout adult life. Nat Neurosci, 2007; 10: 1538-1543

21) Strecker J K, Minnerup J, Schutte-Nutgen K, Gess B, Schabitz W R, Schilling M. Monocyte chemoattractant protein-1-deficiency results in altered blood-brain barrier breakdown after experimental stroke. Stroke, 2013; 44: 2536-2544

22) Schulz C, Gomez Perdiguero E, Chorro L, Szabo-Rogers H, Cagnard N, Kierdorf K, Prinz M, Wu B, Jacobsen S E, Pollard J W, Frampton J, Liu K J, Geissmann F. A lineage of myeloid cells independent of Myb and hematopoietic stem cells. Science, 2012; 336: 86-90

23) Geissmann F, Jung S, Littman D R. Blood monocytes consist of two principal subsets with distinct migratory properties. Immunity, 2003; 19: 71-82

24) Geissmann F, Manz M G, Jung $S$, Sieweke M H, Merad M, Ley K. Development of monocytes, macrophages, and dendritic cells. Science, 2010; 327: 656-661

25) Qiao J H, Tripathi J, Mishra N K, Cai Y, Tripathi S, Wang X P, Imes S, Fishbein M C, Clinton S K, Libby P, Lusis A J, Rajavashisth T B. Role of macrophage colonystimulating factor in atherosclerosis: studies of osteopetrotic mice. Am J Pathol, 1997; 150: 1687-1699

26) Haeusler K G, Schmidt W U, Föhring F, Meisel C, Helms T, Jungehulsing G J, Nolte C H, Schmolke K, Wegner B, Meisel A, Dirnagl U, Villringer A, Volk H D. Cellular immunodepression preceding infectious complications after acute ischemic stroke in humans. Cerebrovasc Dis, 2008; $25:$ 50-58

27) Liberale L, Montecucco F, Bonaventura A, Casetta I, Seraceni S, Trentini A, Padroni M, Dallegri F, Fainardi E, Carbone F. Monocyte count at onset predicts poststroke outcomes during a 90-day follow-up. Eur J Clin Invest, 2017; 47: 702-710

28) Viedt C, Orth S R. Monocyte chemoattractant protein-1 (MCP-1) in the kidney: does it more than simply attract monocytes? Nephrol Dial Transplant, 2002; 17: 20432047

29) Murphy A J, Woollard K J. High-density lipoprotein: a potent inhibitor of inflammation. Clin Exp Pharmacol Physiol, 2010; 37: 710-718 
30) Sanossian N, Tarlov N E. HDL-C and LDL-C: their role in stroke pathogenesis and implications for treatment. Curr Treat Options Cardiovasc Med, 2008; 10: 195-206

31) Ansell B J, Navab M, Hama S, Kamranpour N, Fonarow G, Hough G, Rahmani S, Mottahedeh R, Dave R, Reddy $S$ T, Fogelman A M. Inflammatory/antiinflammatory properties of high-density lipoprotein distinguish patients from contro 1 subjects better than high-density lipoprotein cholesterol levels and are favorably affected by sim vastatin treatment. Circulation, 2003; 108: 2751-2756

32) Worthmann H, Dengler R, Schumacher H, Schwartz A, Eisert W G, Lichtinghagen R, Weissenborn K. Monocyte chemotactic protein-1 as a potential biomarker for early anti-thrombotic therapy after ischemic stroke. Int J Mol Sci, 2012; 13: 8670-8678

33) Kanbay M, Solak $Y$, Unal H U, Kurt Y G, Gok M, Cetinkaya H, Karaman M, Oguz Y, Eyileten T, Vural A, Covic A, Goldsmith D, Turak O, Yilmaz M I. Monocyte count/HDL cholesterol ratio and cardiovascular events in patients with chronic kidney disease. Int Urol Nephrol, 2014; 46: 1619-1625

34) Norman J E, Tong S. MHR welcomes high-quality basic reproductive research around pregnancy. Mol Hum Reprod, 2013; 19: 709-710

35) Ziaee M, Javanmard D, Sharifzadeh G, Hasan Namaei M, Azarkar G. Genotyping and Mutation Pattern in the Overlapping MHR Region of HBV Isolates in Southern
Khorasan, Eastern Iran. Hepat Mon, 2016; 16: e37806

36) Canpolat U, Aytemir K, Yorgun H, Ahiner L, Kaya E B, Ay S, Topaloğlu S, Aras D, Oto A. The role of preprocedural monocyte-to-high-density lipoprotein ratio in prediction of atrial fibrilla tion recurrence after cryoballoonbased catheter ablation. Europace, 2015; 17: 1807-1815

37) Cetin M S, Ozcan Cetin E H, Kalender E, Aydin S, Topaloglu S, Kisacik H L, Temizhan A. Monocyte to HDL Cholesterol Ratio Predicts Coronary Artery Disease Severity and Future Major Cardiova scular Adverse Events in Acute Coronary Syndrome. Heart Lung Circ, 2016; 25: 1077-1086

38) Lehmann M F, Kallaur A P, Oliveira S R, Alfieri D F, Delongui F, De Sousa Parreira J, De Araujo M C, Rossato C, De Almeida J T, Pelegrino L M, Bragato E F, Lehmann A L, Morimoto H K, Lozovoy M A, Simao A N, KaimenMaciel D R, Reiche E M. Inflammatory and metabolic markers and short-time outcome in patients with acute ischemic stroke in relation to TOAST subtypes. Metab Brain Dis, 2015; 30: 1417-1428

39) S G, Am G, M R, Sl A, Ae B, M B, Physiology S a J J O C. Monocyte-to-HDL-cholesterol ratio as a prognostic marker in cardiovascular diseases. 2018; 233: 9237-9246

40) M Y, Medicine K H J J O C. A New Inflammatory Marker: Elevated Monocyte to HDL Cholesterol Ratio Associated with Smoking. 2018; 7: undefined 
Supplemental Table 1. Baseline characteristics of study patients with acute ischemic stroke

\begin{tabular}{|c|c|c|c|}
\hline Characteristics & Enrolled $(n=1,090)$ & Excluded $(n=203)$ & $P$ Value \\
\hline Age, y & $60.09 \pm 12.78$ & $60.42 \pm 11.80$ & 0.803 \\
\hline Male & $714(62.9 \%)$ & $61(54.5 \%)$ & 0.074 \\
\hline Smoking & $417(38.3 \%)$ & $65(32.2 \%)$ & 0.217 \\
\hline Statin use history & $66(6.1 \%)$ & $18(8.9 \%)$ & 0.668 \\
\hline NIHSS score at admission & $3(2-6)$ & $3(1-6)$ & 0.868 \\
\hline Hypertension & $604(53.3 \%)$ & $71(63.4 \%)$ & 0.030 \\
\hline $\mathrm{CHD}$ & $119(10.5 \%)$ & $22(12.7 \%)$ & 0.059 \\
\hline Atrial fibrillation & $57(5 \%)$ & $14(8.5 \%)$ & 0.148 \\
\hline Diabetes mellitus & $198(17.5 \%)$ & $299(18.3 \%)$ & 0.610 \\
\hline Stroke & $209(18.4 \%)$ & $30(18.9 \%)$ & 0.702 \\
\hline Dyslipidemia & $78(6.9 \%)$ & $11(6.5)$ & 0.633 \\
\hline WBC, $10^{9} / \mathrm{L}$ & $7.51(6.31-8.51)$ & $7.61(6.13-9.67)$ & 0.608 \\
\hline Neutrophil, $10^{9} / \mathrm{L}$ & $5.10(3.91-6.11)$ & $5.15(3.88-7.21)$ & 0.471 \\
\hline Lymphocyte, $10^{9} / \mathrm{L}$ & $1.71(1.31-2.11)$ & $1.65(0.95-2.15)$ & 0.638 \\
\hline MONO, $10^{9} / \mathrm{L}$ & $0.56(0.48-0.63)$ & $0.61(0.45-0.79)$ & 0.333 \\
\hline $\mathrm{CHO}, \mathrm{mmol} / \mathrm{L}$ & $4.07(3.46-4.77)$ & $4.16(3.11-4.70)$ & 0.571 \\
\hline $\mathrm{TG}, \mathrm{mmol} / \mathrm{L}$ & $1.37(1.01-2.01)$ & $1.35(0.91-1.89)$ & 0.309 \\
\hline LDL-C, mmol/L & $2.49(1.93-3.06)$ & $2.63(1.75-3.18)$ & 0.742 \\
\hline HDL-C, mmol/L & $1.06(0.91-1.24)$ & $1.06(0.89-1.33)$ & 0.923 \\
\hline MHR & $0.48(0.35-0.61)$ & $0.46(0.34-0.64)$ & 0.934 \\
\hline
\end{tabular}

NIHSS, National Institutes of Health Stroke Scale; CHD, coronary heart disease; WBC, white blood cell, MONO, monocyte; CHO, total cholesterol; TG, triglyceride; LDL-C, low-density lipoprotein; HDL-C, high-density lipoprotein and MHR, monocyte to high-density lipoprotein ratio.

Supplemental Table 2. Multivariate logistic regression analysis predicting the 3-month outcomes

\begin{tabular}{|c|c|c|c|c|}
\hline \multirow[b]{2}{*}{ Model } & \multirow[b]{2}{*}{ Independent variable } & \multicolumn{3}{|c|}{ Multivariate logistic regression analysis ${ }^{*}$} \\
\hline & & OR & $95 \%$ CI & $P$ Value \\
\hline \multirow{14}{*}{$\begin{array}{l}\text { Model } 1 \\
\text { (with MONO } \\
\text { and HDL-C) }\end{array}$} & Age, y & 1.03 & $1.02-1.05$ & 0.001 \\
\hline & Male & 1.66 & $1.13-2.43$ & 0.010 \\
\hline & Smoking & 1.34 & $1.03-1.71$ & 0.026 \\
\hline & Statin use history & 1.58 & $1.01-2.54$ & 0.042 \\
\hline & NIHSS score at Admission & 1.78 & $1.14-2.78$ & 0.011 \\
\hline & History of hypertension & 1.18 & $0.81-1.74$ & 0.388 \\
\hline & History of atrial fibrillation & 1.40 & $0.72-2.69$ & 0.320 \\
\hline & History of stroke & 1.52 & $1.02-2.27$ & 0.039 \\
\hline & History of CHD & 1.29 & $0.78-2.14$ & 0.318 \\
\hline & History of diabetes mellitus & 1.17 & $0.76-1.78$ & 0.477 \\
\hline & Neutrophil, $10^{9} / \mathrm{L}$ & 1.11 & $1.02-1.20$ & 0.016 \\
\hline & $\mathrm{TG}, \mathrm{mmol} / \mathrm{L}$ & 0.81 & $0.65-1.01$ & 0.057 \\
\hline & MONO, $10^{9} / \mathrm{L}$ & 2.21 & $1.05-4.68$ & 0.038 \\
\hline & HDL-C, mmol/L & 0.56 & $0.28-1.09$ & 0.088 \\
\hline \multirow{13}{*}{$\begin{array}{l}\text { Model } 2 \\
\text { (with MHR) }\end{array}$} & Age, $y$ & 1.03 & $1.01-1.04$ & 0.001 \\
\hline & Male & 1.38 & $0.96-1.99$ & 0.084 \\
\hline & Smoking & 1.27 & $1.01-1.65$ & 0.016 \\
\hline & Statin use history & 1.71 & $1.11-2.34$ & 0.049 \\
\hline & NIHSS score at Admission & 1.68 & $1.07-2.63$ & 0.024 \\
\hline & History of hypertension & 1.20 & $0.84-1.73$ & 0.314 \\
\hline & History of atrial fibrillation & 1.38 & $0.72-2.62$ & 0.332 \\
\hline & History of stroke & 1.30 & $0.89-1.90$ & 0.184 \\
\hline & History of CHD & 1.20 & $0.74-1.96$ & 0.461 \\
\hline & History of diabetes mellitus & 1.25 & $0.84-1.87$ & 0.266 \\
\hline & Neutrophil, $10^{9} / \mathrm{L}$ & 1.17 & $1.09-1.25$ & 0.001 \\
\hline & $\mathrm{TG}, \mathrm{mmol} / \mathrm{L}$ & 0.81 & $0.66-0.99$ & 0.043 \\
\hline & MHR & 2.58 & $1.21-5.51$ & 0.015 \\
\hline
\end{tabular}

NIHSS, National Institutes of Health Stroke Scale; CHD, coronary heart disease; WBC, white blood cell, MONO, monocyte; CHO, total cholesterol; TG, triglyceride; LDL-C, low-density lipoprotein; HDL-C, high-density lipoprotein and MHR, monocyte to high-density lipoprotein ratio. 
Supplemental Table 3. Odds ratios and 95\% confidence intervals of primary and secondary outcomes associated with MHR, as a categorical variable, after acute ischemic stroke onset

\begin{tabular}{|c|c|c|c|c|c|}
\hline \multirow[b]{2}{*}{ Outcomes } & \multicolumn{5}{|c|}{ MHR } \\
\hline & $\begin{array}{c}\text { Quartile } 1 \\
<0.345\end{array}$ & $\begin{array}{c}\text { Quartile 2 } \\
0.345-0.451\end{array}$ & $\begin{array}{c}\text { Quartile } 3 \\
0.451-0.602\end{array}$ & $\begin{array}{c}\text { Quartile } 4 \\
\geq 0.602\end{array}$ & $P$ Trend \\
\hline \multicolumn{6}{|l|}{ Primary outcome } \\
\hline \multicolumn{6}{|l|}{ Death and major disability (mRS, 3-6) } \\
\hline No. of cases (\%) & $41(14.5 \%)$ & $35(12.3 \%)$ & $49(17.3 \%)$ & $62(21.8 \%)$ & \\
\hline Multivariable adjusted model ${ }^{*}$ & 1.00 & $0.82(0.51-1.34)$ & $1.23(0.78-1.94)$ & $1.64(1.06-2.54)$ & 0.016 \\
\hline \multicolumn{6}{|l|}{ Secondary outcomes } \\
\hline \multicolumn{6}{|l|}{ Death } \\
\hline No. of cases $(\%)$ & $6(2.1 \%)$ & $7(2.4 \%)$ & $5(1.8 \%)$ & $10(3.5 \%)$ & \\
\hline Multivariable adjusted model & 1.00 & $1.22(0.49-2.99)$ & $1.58(0.67-3.71)$ & $1.97(0.49-2.99)$ & 0.028 \\
\hline \multicolumn{6}{|l|}{ Death or disability (mRS, 2-6) } \\
\hline No. of cases (\%) & $88(24.2 \%)$ & $76(20.9 \%)$ & $94(25.8 \%)$ & $106(29.1 \%)$ & \\
\hline Multivariable adjusted model & 1.00 & $0.73(0.51-1.04)$ & $1.19(0.84-1.68)$ & $3.34(1.56-4.61)$ & 0.033 \\
\hline \multicolumn{6}{|l|}{ Modified Rankin Scale** } \\
\hline 0 (no symptoms) & $118(41.4 \%)$ & $125(44.1 \%)$ & $109(38.4 \%)$ & $112(39.2 \%)$ & \\
\hline 1 (no significant disability despite symptoms) & $98(34.4 \%)$ & $100(35.1 \%)$ & $102(35.8 \%)$ & $90(31.7 \%)$ & \\
\hline 2 (slight disability) & $28(9.7 \%)$ & $24(8.6 \%)$ & $24(8.5 \%)$ & $21(7.3 \%)$ & \\
\hline 3 (moderate disability) & $17(5.8 \%)$ & $13(4.6 \%)$ & $24(8.4 \%)$ & $29(10.2 \%)$ & \\
\hline 4 (moderately severe disability) & $14(4.9 \%)$ & $9(2.8 \%)$ & $13(4.6 \%)$ & $14(4.9 \%)$ & \\
\hline 5 (severe disability) & $5(1.7 \%)$ & $7(2.5 \%)$ & $7(2.5 \%)$ & $9(3.2 \%)$ & \\
\hline 6 (dead) & $6(2.1 \%)$ & $7(2.4 \%)$ & $5(1.8 \%)$ & $10(3.5 \%)$ & \\
\hline Multivariable adjusted model & 1.00 & $1.43(1.19-1.67)$ & $1.60(1.36-1.84)$ & $1.07(1.03-1.09)$ & 0.125 \\
\hline
\end{tabular}

*Adjustment for age, gender, MONO, HDL-C; NIHSS score at admission, history of stroke, history of atrial fibrillation, history of coronary heart disease, history of hypertension, history of diabetes mellitus, neutrophil, triglyceride, smoking, statin use history;

* Odds of a 1-U higher modified Rankin Scale score MHR, monocyte to high-density lipoprotein ratio. 